\title{
The Predictive Five: A supervised learning approach to personality psychology
}

Gal Lavi (Ben-Gurion University) , Michael Gilead* (Ben-Gurion University), Jonathan D. Rosenblatt*

(Ben-Gurion University)

*equal contribution

In the current study, we set out to examine the viability of a novel approach to modeling human personality. Current research in psychology suggests that people's personalities can be effectively described using five broad dimensions (the Five-Factor Model; FFM); however, the FFM has been criticized for its relatively limited predictive ability. We propose a novel approach to modeling human personality that is based on the maximization of the model's predictive accuracy. Unlike the FFM, which performs unsupervised dimensionality reduction, we utilized supervised machine learning techniques for dimensionality reduction of questionnaire data, using numerous psychologically meaningful outcomes as data labels (e.g., intelligence, well-being, sociability). The results showed that our five dimensional personality summary, which we term the Predictive Five (PF), provides predictive performance that is superior to the FFM in independent validation datasets, and on a new set of outcome variables selected by an independent group of psychologists. Furthermore, we examine the between-participants' replicability of the PF representation and show that the PF has good test-retest reliability, and as such provides an important addition to the psychologists' toolbox. The approach described herein has the promise of providing an interpretable low-dimensional personality representation, which is also predictive of behaviour.

\section{Introduction}

Humans significantly differ from each other. Some people's idea of fun is partying all night long, and others enjoy binging on a TV series while eating snacks; some are extremely intelligent, and others less so; some are hot-headed, and others remain cool, no matter what. Because of this variety, predicting humans' thoughts, feelings, and behaviors is a 
cumbersome task; nonetheless, this task is unavoidable. For example, when we decide who to marry, we try to predict whether we can depend on the other person till death do us part; when we choose a career, we must do our best to predict whether we will be successful and fulfilled in a given profession.

In order to predict a person's thoughts, feelings, and behaviors, people often have no other option but to generate something akin to a scientific theory (Newcomb \& Heider 1958)--a parsimonious model that attempts to capture the unique characteristics of individuals, and that could be used to predict their behavior in novel circumstances. Indeed, research shows that people employ such theories when predicting their own (e.g., Dweck, 2013) and others' (e.g., Newmann, 1996; Thornton \& Tamir, 2017) behaviors. Unfortunately, theories based strictly on intuition are often highly inaccurate (Swann, 1984), even if produced by professional psychological theoreticians (Ægisdóttir et al., 2006). In light of this, ever since the early days of psychology research, scholars have been attempting to devise personality models using the scientific method, giving rise to the longstanding field of personality research.

Personality, when used as a scientific term, refers to the mental features of individuals that characterize them across different situations, and thus can be used to predict their behavior. In the early years of personality research, scientists generated numerous competing theories and measures, but struggled to arrive at a scientific consensus regarding the core structure of human personality. Early attempts at theoretical integration suggested that two constructs termed neuroticism and extraversion (the so-called "Big Two"; Wiggins, 1968) capture much of the variance in people's psychological makeup. Further research analyzing psychological questionnaire data suggested the existence of a third crucial dimension, termed openness to experience, giving rise to the NEO (Neuroticism, Extraversion, Openness) model of personality. In recent decades, this three-factor model was replaced by a consensus theory of the core dimensions of human personality—the Five Factor Model (FFM). 
The FMM emerged from the so-called "lexical paradigm", which assumed that if people regularly exhibit a form of behavior that is meaningful to human life, then language will produce a term to describe it (Allport \& Odbert, 1936). Given this assumption, personality psychologists performed research wherein they asked individuals to rate themselves on lists of language-derived trait words (e.g., friendly, upbeat), and then developed and used early dimensionality-reduction methods to find a parsimonious model that can account for much of the variability in each person's trait ratings (e.g., Cattell, 1943). The research that stemmed from the lexical hypothesis identified the three constructs of the NEO model, as well as two additional constructs termed Conscientiousness and Agreeableness (e.g., McCrae \& Costa, 1989).

Much research shows that these five factors, often termed the "Big Five" are relatively stable over time and have convergent and discriminant validity across methods and observers _(Costa \& McCrae, 1990). Moreover, research into the FFM has replicated the dimensional structure in different samples, languages, and cultures (Allik \& Allik 2002; Benet-Martínez \& John, 1998). In light of this, the FFM is taken by some to reflect a comprehensive ontology of the psychological makeup of human beings (John, 1990a); according to McCrae and Costa (1986), the five factors are "both necessary and reasonably sufficient for describing at a global level the major features of personality".

Surely, human beings are complex entities, and their personality is not fully captured by five dimensions; however, the importance of having a parsimonious model of humans' psychological diversity cannot be overstated. As noted by John and Srivastava (1999), a parsimonious taxonomy permits researchers to study "specified domains of personality characteristics, rather than examining separately the thousands of particular attributes that make human beings individual and unique". Moreover, as noted by John and Srivastava, such a taxonomy greatly facilitates "the accumulation and communication of empirical findings by offering a standard vocabulary, or nomenclature."

An additional consequence of having a parsimonious model of the core dimensions of human personality, is that such an abstraction enables the acquisition of novel 
knowledge via statistical learning (see Gilead, Liberman, \& Trope, 2020, for a discussion of the importance of abstract representations in learning); namely, whereas the estimation of covariances between high-dimensional vectors is often highly unreliable (i.e., the so-called curse of dimensionality", e.g., Fukunaga, 1990), learning the statistical correlates of a low-dimensional structure is a relatively tractable problem. For example, research has shown that the participants' self-reported ratings on the superordinate FFM dimensions can be reliably estimated based on their digital footprint (Kosinski et al., 2013).

This ability to infer individuals' personality traits using machine learning also raises serious concerns, as it may be used for effective psychological manipulation of the public. In 2013, a private company named Cambridge Analytica harvested the data of hundreds of millions of Facebook users, and used statistical methods to infer the personality characteristics of hundreds of millions of Americans ${ }^{1}$. This psychological profile of the American population was supposedly used by the Trump campaign in an attempt to tailor political advertisements based on an individuals' specific personality profile. While the success of these methods remains unclear, given the vast amount of data accumulated by companies such as Google and Facebook, the potential dangers of machine-learning based psychological profiling is taken by many to be a serious threat to democracy (e.g., Noah Harari, 2015).

Even if dubious entities indeed manage to acquire the Big Five personality profile of entire populations, it is far from obvious that such information could be used to generate actionable predictions. Indeed, the FFM was often criticized for its limited contribution to predicting outcomes on meaningful dimensions (Hough, 1992; e.g., workplace performance, Salgado, 2002; political affiliation, Sibley et al., 2012). As Hough (1992) harshly concluded, "if prediction of life outcomes or criteria is important in evaluating personality taxonomies, the Big Five is an inadequate taxonomy". Given the limited relations between real-world consequences and the FFM, it has also been suggested that

\footnotetext{
${ }^{1}$ https://www.nytimes.com/2018/04/04/us/politics/cambridge-analytica-scandal-fallout.html
} 
the public concern over the Cambridge Analytica scandal was overblown (e.g., Gibney, 2018).

Moreover, the low predictive validity of personality measures was taken by some as an indication that human endeavours are not driven by some core psychological makeup (e.g., Mischel, 1968). However, the fact that the predictive validity of the FFM is sub-optimal, does not necessarily entail that personality is inconsequential. Instead, it may simply reflect the fact that the statistical method by which the FFM was produced--namely, factor analysis--is not geared towards prediction.

Factor analysis is an unsupervised dimensionality-reduction ${ }^{2}$ method aimed at maximizing explanatory coherence and semantic interpretability, rather than maximizing predictive ability. It does so by finding a parsimonious, low-dimension representation (e.g., the Big Five factors) that maximizes the variance explained in the higher-dimension domain (e.g., hundreds of responses to questionnaire items). Advances in statistical machine learning have opened up new techniques for supervised dimensionality-reduction. Namely, methods that reduce the dimensionality of a source domain (i.e., predictor variables, $X_{1}, \ldots, X_{n}$; in the case of personality, hundreds of questionnaire items) by focusing on the objective of maximizing the capacity of the lower-dimensional representation to predict outcomes of a target domain (e.g., outcome variables, $Y_{1}, \ldots, Y_{m}$ ).

Such novel techniques wherein dimensionality-reduction are achieved via maximization of predictive accuracy across a host of target-domain outcomes hold the potential of providing psychologists with parsimonious models of a psychological feature space that serve as "universal predictors" of human behavior. Moreover, it may demonstrate that privacy leaks, a-lá Cambridge-Analytica, are indeed a serious threat to democracy, despite being currently dismissed as science fiction (e.g., Gibney, 2018).

${ }^{2}$ Unsupervised dimensionality-reduction refers to the methods that project the original data to a new lower dimensional space without utilizing the outcomes information. 
In light of this, we investigated whether a supervised dimensionality-reduction approach that takes into account a host of meaningful outcomes can outperform the unsupervised methods, which were used to build the Five Factor model, in terms of allowing prediction of humans' behaviors, cognitions, and emotions. Such an approach could pave the way to an entirely new family of personality models and could advance the study of personality. However, alternatively, it may be the case that the FFM indeed "carves nature at its joints" and provides the most accurate ontology of the psychological proclivities of humans. In such a case, the FFM may remain the best predictive model of personality, and our approach will not provide improvements in predictions.

In order to examine this question, we conducted four studies. In Study 1, we built a supervised learning model using big data of personality questionnaire items and important life outcomes. We validated our personality model on held-out data and examined its performance compared to the Big Five model. Next, in Studies 2 and 3, we explored the generalizability of the model to new outcome variables, which the model was not trained on. Finally, Study 4, investigated the ontological status of our model by examining the reproducibility of the model and its test-retest reliability.

\section{Study 1}

\section{Method}

Participants. The analyses relied on the myPersonality dataset that was collected between 2007-2012 via the myPersonality Facebook application. The application enabled its users to take various validated psychological and psychometric tests, such as different versions of the International Personality Item Pool (IPIP) questionnaire. Participation was free of charge, and some of the users were motivated by financial rewards or credit points (Kosinski et al., 2015). All participants provided informed consent for the data they provided to be used in subsequent psychological studies. We used data from 397,851 participants (210,279 females, 142,497 males, and 44,805 did not identify) who answered all of the questions on the 100-item IPIP representation of Goldberg's (1992) markers for 
the Big Five factor structure. Participants' mean age was 25.7 years $(S D=8.84)$. The study was approved by the Institutional Review Board of Ben-Gurion University of the Negev.

\section{Measures.}

Dependent Variables. We sought to use supervised learning in order to find a low-dimensional representation of personality that can be used to predict psychological consequences across a diverse set of domains. We thus focused on ten meaningful outcome variables that were available in the myPersonality database, which we considered to be representative of many important aspects of human life:

(1) IQ, scores, measured with 20 IQ items (Stillwell \& Kosinski, 2015).

(2) Well-being, measured with the Satisfaction With Life Score (Diener et al., 1985).

Personal values, measured using two scores representing the two axes from the Schwartz's Values Survey: (3) Self-transcendence vs. Self-enhancement values and (4) Openness to Change vs. Conservation values (Schwartz, 1992).

(5) Empathy, measured with the Empathy Quotient Scale (Baron-Cohen \& Wheelwright, 2004).

(6) Depression, measured with The Center for Epidemiologic Study Depression Scale (Radloff, 1977).

(7) Risky behavior, measured with a single-item question concerning illegal drug use.

(8) Self-reports of legal, yet unhealthy behavior (i.e., alcohol and smoking consumption).

(9) Self-report of political ideology.

(10) The number of friends of participants' had on the social network Facebook.

Independent Variables. As part of the 100-IPIP questionnaire, the subjects were asked to rate their agreement with various statements related to different behaviors in 
their life and their general characteristics and competencies, on a scale from 1 (strongly disagree) to 5 (strongly agree).

Statistical Method. The problem we set out to solve is to find a good predictive model that is: (a) based on the 100 variables of the existing IPIP questionnaire, and (b) uses five variables only, so we can fairly compare it with the FFM. Reduced Rank Regression (RRR) is a tool that allows just that: it compresses the original 100 IPIP items, to a set of five new variables. These new variables are constructed so that they are good predictors, on average, of a large set of outcomes. Unlike PCA or Factor Analysis, this method reduces data dimensionality by optimizing predictive accuracy.

We randomly divided our data into an independent train and test sets. Each subject in the train and test set had 100 scores of the IPIP questionnaire $\left(X_{1}, X_{2}, \ldots, X_{100}\right)$, as well as their score in each of the ten dependent variables $\left(Y_{1}, Y_{2}, \ldots, Y_{10}\right)$.

Assume $X(n \times 100)$ and $Y(n \times 10)$ have been centered. We fitted a linear predictor, with coefficient vector:

$$
\text { (1) } \hat{Y}_{j}:=\sum_{k=1}^{100} X_{k} C_{k j}, \quad j=1,2, \ldots .10
$$

And in matrix notation:

$$
\text { (2) } \hat{Y}=X C
$$

Our linear predictors were fully characterized by the matrix C. We wanted these predictors to satisfy the following criteria: (a) They minimize the squared prediction loss. (b) They consist of 5 predictors, i.e., $\operatorname{rank}(C)=r=5$. Criterion (a) ensures the goodness of fit of the model, and criterion (b) ensures a fair comparison with the Big Five. The RRR problem amounts to finding a set of predictors, $\hat{C}$, so that

$$
\text { (3) } \widehat{C}:=\operatorname{argmin}_{C}\left\{\|Y-X C\|^{2} \text {, such that } \operatorname{rank}(C)=r\right\} \text {, }
$$


Where $\|\cdot\|$ denotes the Frobenius matrix norm. The matrix $C$ can be expressed as a product of two rank constrained matrices:

$$
\text { (4) } C:=B A^{T}
$$

Where $B$ is of dimension $p \times r$ and $A$ is of dimension $q \times r$. The model (2) may thus be rewritten as:

$$
\text { (5) } \hat{Y}=(X \hat{B}) \hat{A}^{T}
$$

The $n \times r$ matrix $X \hat{B}$, which we noted $\tilde{X}$, may be interpreted as our new low dimensional set of personality attributes. Crucially for our purposes, the same set of $r$ predictors is used for all dependent variables. By choosing dependent variables from various different domains, we dare argue that this set of predictors can serve as a set of "universal predictors", which we call henceforth the Predictive Five (PF). For the details of the estimation of $\hat{B}$ for example see Chen et al. (2012).

Model assessment. To assess the predictive performance of the Predictive Five, and compare it to the predictive properties of the classical FFM, we used a 4-fold cross validation scheme. The validation worked as follows: we learned ${ }^{3} \hat{B}$ from the general train set (397,851 samples) using RRR method; we divided the independent test set (800 samples) into 4 subsets; we learned $\hat{A}$ from a three-quarters part of the test set (600 samples), and computed the $R^{2}$ on the holdout test set (200 samples); we iterated this process over the 4-test subsets. The rationale of this scheme is that: (a) Predictive performance is assessed using $R^{2}$ on a completely novel dataset; (b) When learning the predictive model, we wanted to treat the personality attributes as known. We thus learned $\hat{B}$ and $\hat{A}$ from different sets. The size of the holdout set was selected so that $R^{2}$ estimates will have low variance. The details of the process can be found in the accompanying code.

\footnotetext{
3 "Learning" is the machine-learning equivalent of "estimating" or "fitting" in statistical parlance.
} 
Another candidate reference model is Principal Component Regression (PCR), where we reduced the IPIP questionnaire to its 5 leading principal components, which we call the PCR Five [2]. We considered the PC Five as another set of personality attributes, which we used in a follow-up regression. Like the RRR case, we learned the PC Five from the train-set (397,851 samples). Next we divided the independent test set (800 samples) into 4 subsets and used a 4-fold cross validation scheme to learned the predictive model and to validated it by computing $R^{2}$ on a fresh holdout dataset.

\section{Results}

Factor Loadings. Each of the resulting five factors was a weighted linear combination of item responses, measuring the five different dimensions of the FFM. Despite the fact that the resulting model was based on a questionnaire meant to reliably gauge the FFM, the resulting outcome did not fully reproduce the five factor structure. The detailed factor loadings for each of the resulting five can be examined in an online application we have created (https://predictivefive.shinyapps.io/PredictiveFive), and can be easily gleaned by examining the correlation of Predictive Five scores to the Big Five (Figure 1).

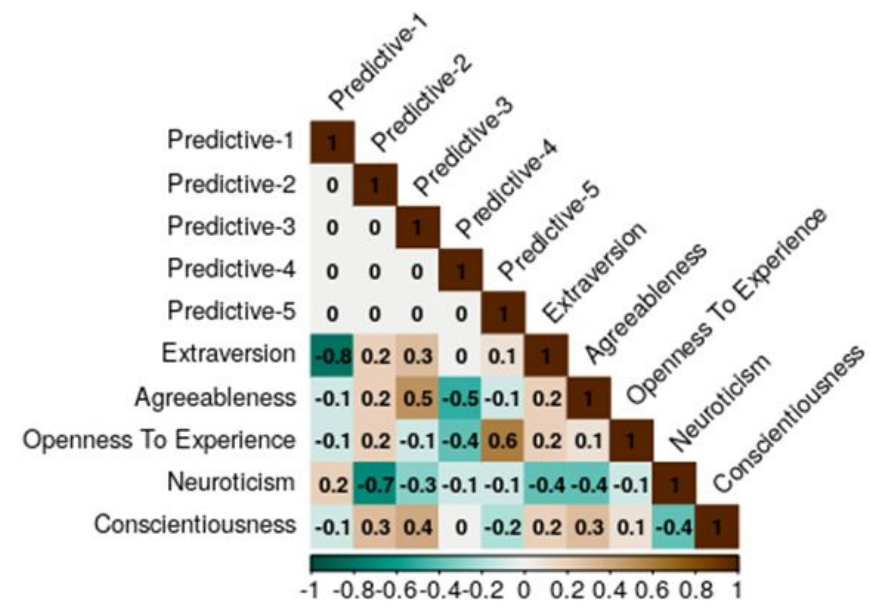

Figure 1. Correlations between the Predictive Five factors and FFM factors. 
As can be seen from Figure 1, predictive factors 1 and 2 are correlated with Extraversion and Neuroticism, respectively, and Predictive Factor 5 is related to Openness. However, factors 3 and 4 reflect a relatively even combination of several FFM dimensions.

Model Assessment. The out-of-sample $R^{2}$ of the three models (with the PF $\backslash$ the Big Five $\backslash$ the PC Five) is reported in Figure 2. From this figure, we learn that the PF dimensions are better predictors of the outcome variables. This holds true on average (over behavioral outcomes), but also for each outcome individually. The average (over outcomes) $R^{2}$ of the PF was $22 \%$, compared to $14.7 \%$ for the Big Five, and $16.2 \%$ for the PC Five. The variable-wise performance is reported in Table 1. From these we learn that PF is a better predictor than the Big Five on all outcomes: The improvement in the test $R^{2}$ between PF to Big Five varied from a 4.2\% improvement for the Unhealthy Behavior value, to a $208 \%$ improvement for predicting Intelligence.

When comparing the performance of PF to PC model, we see that the performance of the PC model is more similar to FFM than to PF. In particular, the PF outperforms the PC dimensions in eight out of ten outcome variables, sometimes with a considerable margin. 


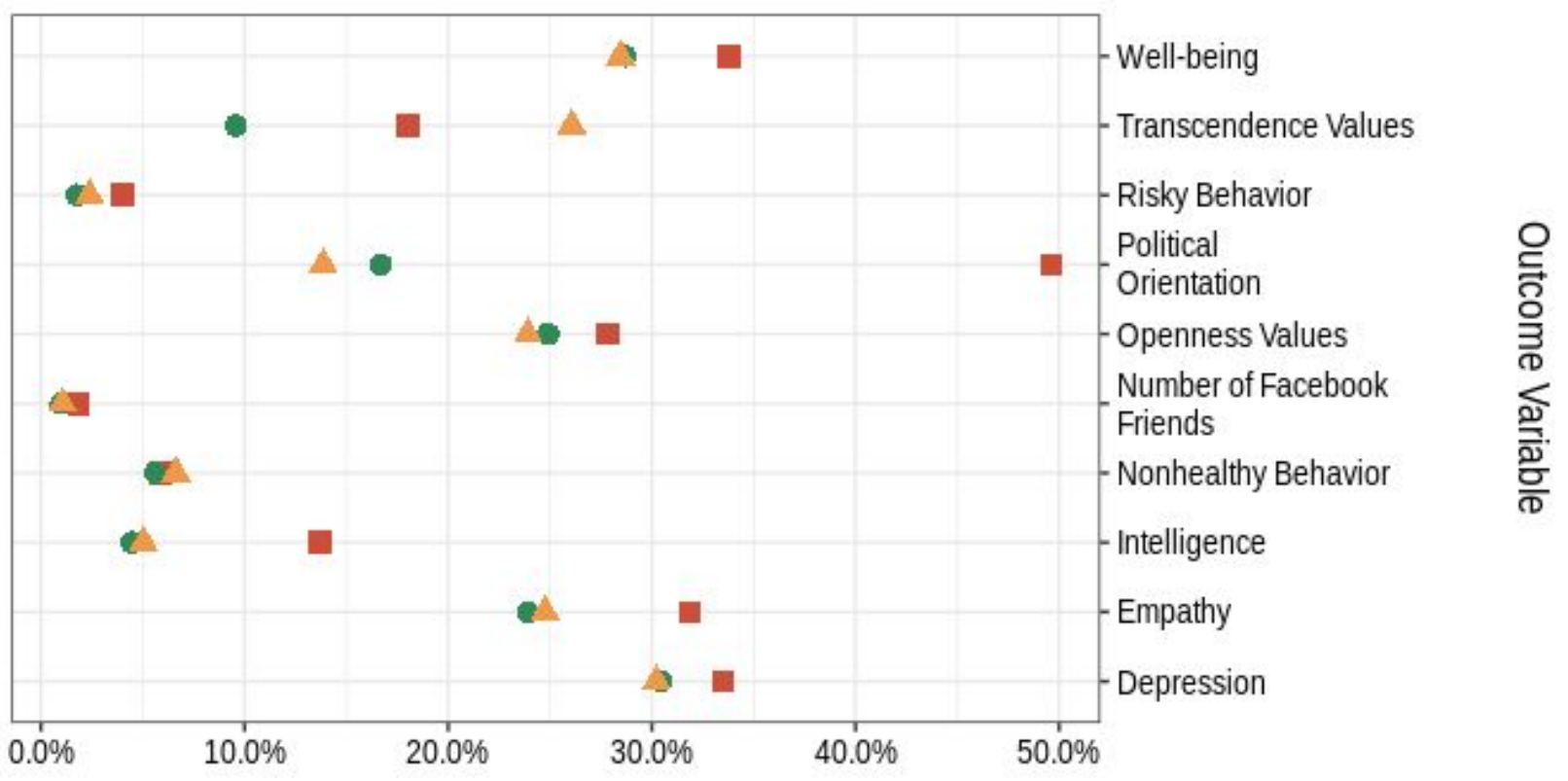

Explained Variance

Predictors - Big Five $\Delta$ PC Five $\square$ Predictive Five

Figure 2. Cross-validated $\mathrm{R}^{2}$ of the ten dependent variables in Study 1. Each model is color and shape coded.

\section{Discussion}

The results of Study 1 demonstrate that by using a standard personality questionnaire and supervised learning methods, it is possible to improve the prediction of psychological outcomes. The RRR algorithm allowed us to compress the 100 questions of the personality questionnaire to a new quintet of attributes that optimize prediction across a diverse set of psychological outcomes. This set of five latent factors $(\tilde{X})$ differs from the FFM, and has better predictive power on the held-out sample than the classical Big Five and PC dimensions.

A theory of personality should strive to predict humans' thoughts, feelings, and behaviors across different life contexts. Indeed, the representation we discovered in Study 1 was superior to the FFM in terms of its ability to predict a diverse set of psychological 
outcomes on a set of novel observations. The fact that the same low-dimensional representation was applicable across many different outcome measures suggest that it is a generalizable model. However, despite the diversity of the outcome measures examined in Study 1, it remains possible that the Predictive Five model is only effective for the prediction of the set of outcome measures on which it was trained. In light of this, in Study 2 we sought to examine the performance of our model on a novel outcome measure that was present in the MyPersonality database, but that was held-out from the model generation process. Specifically, in this study we sought to see whether the Predictive Five model outperforms the FFM in its ability to predict the degree to which participants' parents treated them in a warm (vs. rejecting) manner.

Unlike the outcome measures used in Study 1, this DV does not pertain to participants' lives in the present, rather, it is a measure of their past experiences. As such, "retrodiction" of remote history may be especially challenging. Nonetheless, it is widely held that individuals' personality is shaped, at least to some extent, by the extent to which they were raised in a loving household (e.g., Winnicott, 1973; Bowlby, 1988). As such, per theory, a good personality model should likely contain information that can be used to predict the extent to which person's early family environment was warm and accepting.

\section{Study 2}

\section{Method}

Participants. We used data from 3,869 participants who answered all of the questions on the 100-item IPIP representation of Goldberg's (1992) markers for the Big Five factor structure, and answered the short form EMBU ("My Memories of Upbringing") questionnaire (Arrindell et al., 1999).

\section{Measures}

The short form of the EMBU includes three subscales (warmth, rejection, and overprotection), both measured for two parents. We calculated the extent to which 
participants experienced a warm and accepting household by adding the two warmth scores for each participant from the two rejection scores. This measure had acceptable reliability (cronbach's alpha $=.78$ ).

\section{Results}

The out-of-sample $R^{2}$ of the Big Five was $4.78 \%$ and that of the Predictive Five was 8.71\%. Thus, while both models had difficulty predicting parental warmth, the PF was again superior to the FFM, and yielded $82 \%$ improvement in predictive accuracy. These findings further support the idea that the Predictive Five model confers better predictive performance than the FFM.

\section{Discussion}

The results of Study 2 further support the idea that the PF model is generalizable. However, Study 2 again relied on MyPersonality participants, upon which the PF was built. In light of this, in Study 3 we sought to further test the generality of the PF by examining whether it outperforms the FFM on a set of new participants. Furthermore, we wanted to see whether our model can outperform the FFM on a set of new outcome measures selected by an independent group of professional psychologists, blind to our model-generation procedure.

\section{Study 3}

\section{Method}

Participants. We collected new data using Amazon's Mechanical Turk (www.MTurk.com). M-Turk is an online marketplace that enables data collection from a diverse workforce who are paid upon successful completion of each task. Our target sample size was 500 participants, which is double the size of what is considered a standard, adequate sample size in individual differences research (Schönbrodt \& Perugini, 2013). In practice, 582 participants participated in the study, 35 of them were omitted for failing attention checks, leaving 547 participants in the final dataset (243 females and 304 men). 


\section{Measures.}

Dependent Variables. In order to make sure that the PF generalize across different domains of psychological interest, it was important to generate the list of outcome variables in a way that is not biased by our knowledge of the original ten outcome variables on which the PF was designed (i.e., intelligence, well-being, and so on). Therefore, on January 3rd, 2019, we gathered a list of 12 new outcome measures by posting a call on the Facebook group PsychMAP (https://www.facebook.com/groups/psychmap/) asking researchers: "to name psychological outcome measures that you find interesting, important, and that can be measured on M-Turk using a single questionnaire item on a likert scale". The first 12 outcome measures suggested were used as dependent variables in the current study. Participants rated their agreement with 12 statements related to their daily behaviors (1- Strongly Disagree to 7- Strongly Agree). The items were:

(1) "I care deeply about being a good person at heart".

(2) "I value following my heart/intuition over carefully reasoning about problems in my life".

(3) "Other people's pain is very real to me".

(4) "It is important to me to have power over other people".

(5) "I have always been an honest person".

(6) "When someone reveals that s/he is lonely I want to keep my distance from him/her".

(7) "Before an important decision, I ask myself what my parents would think".

(8) "I have math anxiety".

(9) "I am typically very anxious".

(10) "I enjoy playing with fire". 
(11) "I am a hardcore sports fan".

(12) "Politically speaking, I consider myself to be very conservative".

Independent variables. The answers of users to the 100 questions of the IPIP questionnaire, on a scale from 1 (strongly disagree) to 5 (strongly agree).

Model Assessment. Similarly to Study 1, we use a 4-fold cross validation scheme in order to assess the predictive performance of the PF on new data set and outcome variables. Next, we compared it to the predictive performance of the Big Five. The

validation worked as follows: we had $\hat{B}$ from Study 1, we learned $\hat{A}$ from a part of the new sample (400 samples) and computed the $R^{2}$ on the holdout test set (130 samples). In the spirit of the 4-fold CV, we iterated this process over the 4-test sets and calculated the average test $R^{2}$ for each model.

\section{Results}

Similarly to Study 1, the results showed that the predictive performance of the PF was again higher than that of the Big Five. The average test $R^{2}$ of the PF was $26.95 \%$, compared to $21.5 \%$ for the Big Five. The variable-wise performance of each of the predictors is reported in the appendix. From these we learn that the improvement in $R^{2}$ between PF, to Big Five varied from no improvement to a 185.9\% improvement. 


\section{Discussion}

The out-of-sample $R^{2}$ of the two models (PF \Big Five) in Study 3 show a consistent trend with the results presented earlier in Study 1 and Study 2, that is, a higher percentage of explained variance in the models with the PF as predictors. This improvement observed in Study 3 was somewhat more modest than that observed earlier, is nonetheless non-trivial--given that the set of outcome variables was different from the one the PF model was trained on, and given that the PF model was trained on items from questionnaire designed to measure the Big Five. As such, the results of Studies 1-3 clearly demonstrate the generalizability of the PF.

However, for a construct to be considered worthy of its place in psychological theory, there is also a need to demonstrate that it is reproducible and replicable across different individuals and different times. For example, if it were the case that our model discovery process produces different very different factor structures when run on different samples of participants, then the ontological status of the PF will be called into question. Similarly, if the same individuals, tested on different occasions, receive different scores on the PF dimensions, then the "reality" of the Predictive Five representation should likewise be questioned. Therefore, in Study 4a we examined the between-participants' replicability of the PF representation, and in Study $4 \mathrm{~b}$ we examined its test-retest reliability.

\section{Study 4a}

\section{Method}


Participants. We split the train dataset from Study 1 into two datasets; sample A with 198,850 participants and sample B with 198,851 participants.

\section{Model Generation.}

We repeated the same procedures described in Study 1 for each of the two samples. That is, we build two RRR models, and therefore got two rotation matrices ( $B_{1}$ and $B_{2}$ ); multiplying them in the original IPIP-100 transforms the dimension from 100 to 5 (for more details, see Study 1). Next, for each sample, we applied $B_{1}$ and $B_{2}$ and calculated the correlation between the subject's five scores in each transformation, resulting in two correlation vectors which we averaged by factors.

\section{Results}

The averaged correlation between the two subsets of the data can be in Table 3. The results showed that the factor structure observed in one half of the dataset is replicable in the other half, indicating that the PF representation is a structure that consistently emerges across participants.

\section{Study 4b}

\section{Method}

Participants. We used data from 96,682 users of myPersonality application who answered the IPIP questionnaire twice.

Statistical method. We examined the Test-Retest reliability through Pearson's correlation between each of the PF Factors scores in the first and second attempts to answer the IPIP personality questionnaire.

\section{Results}


Pearson's correlation varied from 0.69 for the Predictive Factor 3, to 0.79 for both Predictive Factors 1 and 5, as displayed in Table 3.

\begin{tabular}{|l|c|c|}
\hline & Test-retest correlation & Correlation between replications \\
\hline Predictive Factor 1 & 0.79 & 0.98 \\
\hline Predictive Factor 2 & 0.75 & 0.77 \\
\hline Predictive Factor 3 & 0.69 & 0.70 \\
\hline Predictive Factor 4 & 0.71 & 0.79 \\
\hline Predictive Factor 5 & 0.79 & 0.86 \\
\hline
\end{tabular}

Table 3. Test-Retest Pearson's correlation results.

\section{Discussion}

The results of Study 4a suggest that the PF emerges across different individuals, and the results of Study $4 \mathrm{~b}$ suggest that this factor structure has an adequate test-retest reliability (Terwee et al., 2007). Together, these findings suggest that the PF representation captures some stable low-dimensional structure that exists within the IPIP personality questionnaire, but differs from the FFM.

\section{General Discussion}

In this contribution, we set out to examine the viability of a novel approach to modeling human personality. Unlike the prevailing Five-Factor Model (FFM) of personality, which was developed by relying on unsupervised dimensionality reduction techniques (i.e., factor analysis), we utilized supervised machine learning techniques for dimensionality reduction, using numerous psychologically meaningful outcomes as data labels (e.g., intelligence, well-being, sociability). Whereas the FFM is optimized towards discovering an ontology of superordinate traits that explain most of the variance on self-report measures of psychological traits, our new approach devised a low-dimensional representation of human trait statements that is optimized towards prediction of life outcomes. Indeed, the results showed that our model, which we term the Predictive Five, provides predictive 
performance that is superior to the one achieved by the FFM in independent validation datasets (Study 1), and on a new set of outcome variables, selected independently of the first study (Studies 2-3). Finally, in Study 4, we saw that the PF emerges consistently across time and across individuals, suggesting that it is a stable latent structure, worthy of scientific investigation.

\section{Interpreting the PF}

The factor structure that emerged when using our supervised-dimensionality reduction approach differed from the FFM. Two factors (Factor 1 and 2) largely reproduced the original FFM factors of Extraversion and Neuroticism. Interestingly, these two factors are the ones that were highlighted in early psychological research as the "Big Two" dimensions of personality (Wiggins, 1966). Predictive Factor 5 was also highly related to an existing FFM dimension, namely, Openness to Experience. These three factors (Neuroticism, Extraversion, and Openness) compose the NEO model of personality, which was replaced by the current consensus model of personality, the Five-Factor model.

The third and fourth factors in the model did not correspond to a single FFM trait, but were composed of a mixture of various items. An inspection of the factor loadings suggests that Predictive Factor 4 is related to some sort of a combative attitude, perhaps captured best by the construct of Dominance (e.g., Murray, 1938; Jackson, 1965; Pratto, Sidanius, \& Malle, 1994). The items that loaded highly on this factor related to hostility ("Do not sympathize with others"; "Insult people"), a right-wing political orientation ("Do not vote for liberal political candidates"), and an approach-oriented (e.g., Higgins \& Kruglanski, \& Pierro, 2003) stance ("Get chores done right away"; "Find it easy to get down to work").

Like Predictive Factor 4, Predictive Factor 3 also seemed to capture approach-oriented characteristics (with high loadings for the items "Get chores done right away" and "Find it easy to get down to work"), however, this factor differed from Predictive Factor 4 in that it represented a harmony-seeking phenotype (e.g., Lueng, 2002). The items highly loaded on this factor were those associated with low levels of narcissism ("keep in 
the background", "do not believe I am better than others") but with a stable self-worth ("am pleased with myself"). Additional items that highly loaded on this factor were those that reflect cooperativity ("concerned with others" and "sympathize with others").

These two dimensions may seem like dialectical opposites. Indeed, the item "sympathize with others" strongly loaded on both factors, but with a different sign. However, the additional items that strongly loaded on these two constructs appear to have provided a context that altered the meaning of this item. This is evident in the fact that Factors 3 and 4 are not correlated with each other. A possible speculative interpretation is that the two phenotypes captured by Factor 3 and 4 can be thought of as two strategies that may have been adaptive throughout human evolution. The first, captured by Factor 4 seems to represent aggressive traits that may have been especially useful in the context of inter-group competition and conflict; the second, captured by Factor 3, seems to represent traits that may be associated with intra-group cooperation and peace.

\section{Towards a predictive science of personality}

As noted, the reasons that people seek models of personality are twofold: first, we want models that allow us to understand, discuss and study the differences between people; second, we need these models in order to be able to predict and affect people's choices, feelings and behaviors (Saucier \& Srivastava, 2015). Current approaches to personality modeling succeeded on the former, providing highly comprehensible dimensions of individual differences (e.g., we can easily understand and communicate the contents of the dimension of "Neuroticism" by using this sparse semantic label). However, the ability of the FFM to accurately predict outcomes in people's lives is severely limited (Hough, 1992; Salgado, 2002; Sibley et al., 2012).

The significance of the current work is that it describes a new approach to modeling human personality, that makes the prediction of behaviour an explicit and fundamental goal. Our research shows that supervised dimensionality reduction methods can generate universal, low-dimensional models of personality with improved predictive accuracy. Such 
an approach could complement the unsupervised dimensionality reduction models that have prevailed for decades in personality research.

Aside from providing a general approach for the generation of personality models, the current research also provides a potentially useful instrument for psychologists across different domains of psychological investigation. Our findings suggest that psychologists who are interested in predicting meaningful consequences (e.g., workplace or romantic compatibility) or in optimizing interventions on the basis of individuals' characteristics (e.g., finding out which individuals will best respond to a given therapeutic technique)—should incorporate the PF dimensions in their predictive models. To facilitate such future research, we provide the $\mathrm{R}$ code that calculates the five dimensions based on answers on the freely available IPIP-100 questionnaire (https://github.com/GalBenY/Predictive-Five).

Nonetheless, the PF model described herein is just a first proof of concept of this general approach, and it is likely that future attempts that are untethered to the constraints undertaken in the current study can provide models of greater predictive accuracy. Specifically, in the current research we relied on the IPIP-100, a questionnaire designed by researchers specifically in order to reliability measure the factors of the Big-Five model, and limited ourselves to a five-dimension solution, to allow comparison with the Big-Five. The PF model outperformed the FFM questionnaire despite these constraints. These results provides a very conservative test for the utility of our approach; moreover, the use of an existing, open-access, widely-used questionnaire means that researchers can now easily apply the PF coding scheme alongside with the FFM coding scheme to their data, and compare the utility of the two models in their own specific research domains. Importantly, future attempts to generate universally-predictive models will likely produce even stronger predictive performance if they relax the constraint of finding exactly five dimensions and perform dimensionality-reduction based on the raw data used to generate the FFM itself--namely, the long list of trait adjectives that exist in human language, and that were reduced into the five-dimension of the Big Five. 
Moreover, whereas previous attempts of modeling human personality necessarily limited by their reliance on the subjective products of the human mind (i.e., were predicated on man-made psychological theories, or subjective ratings of trait words), our approach holds the potential of generating personality models that are based on objective inputs whose meaning we do not fully understand. For example, using the approach outlined herein, one could reduce the data of the genome (3 billion base pairs, approximately 750 megabytes of data) into a low-dimensional "bio-psychological profile" that is optimized towards prediction of meaningful psychological outcomes. Once such a low-dimensional representation is discovered, psychological scientists could conduct research aimed at interpreting these latent, biologically-derived factors. As such, the potentially revolutionary consequence of our approach is that it may provide the basis for a biologically-based model of human personality.

A final question concerning predictive models of personality is whether we even want to generate such models, given the potential of their misuse. In the era of social networks and commercial genetic testing, the predictive approach to personality modeling could theoretically lead to models that render human behavior highly predictable. Such models give rise to both ethical concerns (e.g., unethical use by governments and private companies, as in the Cambridge-Analytica scandal ${ }^{4}$ ) and moral qualms (e.g., if behavior becomes highly predictable, what will it mean for notions of free will and personal responsibility?). While these are all valid concerns, we believe that like all other scientific advancements, personality models are tools that can provide a meaningful contribution to human life (e.g., predicting suicide in order to avoid it; predicting which occupation will make a person happiest). As such, the important, inescapable quest towards generating even more effective models that will allow us to predict and intervene in human behavior is only just the beginning.

\footnotetext{
${ }^{4}$ https://www.nytimes.com/2018/04/04/us/politics/cambridge-analytica-scandal-fallout.html
} 


\section{Data Availability:}

The data for Study 1, 3 and 4 rely on the myPersonality database (https://sites.google.com/michalkosinski.com/mypersonality which is an unprecedented big-data repository for psychological research, used in more than a hundred publications. We achieved permission from the owners of the data to use it for the current research - but we do not have their permission to share it for wider use. The data for Study 2 is available upon request. We also share the complete code and the full model with factor loadings.

\section{Appendices:}

\begin{tabular}{|c|c|c|c|c|}
\hline Outcome Variable & $\begin{array}{c}\text { Predictive } \\
\text { Five }\end{array}$ & Big Five & PC Five & $\begin{array}{c}\text { Relative } \\
\text { Improvement }\end{array}$ \\
\hline Intelligence & $13.70 \%$ & $4.45 \%$ & $5 \%$ & $208.3 \%$ \\
\hline Well-being & $33.79 \%$ & $28.68 \%$ & $28.4 \%$ & $17.79 \%$ \\
\hline Depression & $33.46 \%$ & $30.46 \%$ & $30.2 \%$ & $9.8 \%$ \\
\hline Political Orientation & $49.59 \%$ & $16.7 \%$ & $13.86 \%$ & $196.9 \%$ \\
\hline Risky Behavior & $3.98 \%$ & $1.76 \%$ & $2.39 \%$ & $1 \% \%$ \\
\hline Fucebook Friends & $1.86 \%$ & $0.98 \%$ & $5.63 \%$ & \\
\hline
\end{tabular}




\begin{tabular}{|c|c|c|c|c|}
\hline Transcendence Values & $18.04 \%$ & $9.57 \%$ & $26.06 \%$ & $88.52 \%$ \\
\hline Openness Values & $27.85 \%$ & $24.9 \%$ & $23.9 \%$ & $11.82 \%$ \\
\hline Empathy & $31.87 \%$ & $23.92 \%$ & $24.75 \%$ & $33.22 \%$ \\
\hline
\end{tabular}

Table 1. Cross validated test $R^{2}$ of the ten dependent variables in Study 1.

\begin{tabular}{|c|c|c|c|}
\hline & & & \\
& Test Predictive Five & Test Big Five & Predictive Five-Big Five \\
\hline $\begin{array}{c}\text { I care deeply about } \\
\text { being a good person } \\
\text { at heart }\end{array}$ & 0.37442 & 0.322712 & \\
\hline $\begin{array}{c}\text { I value following my } \\
\text { heart/intuition over } \\
\text { carefully reasoning } \\
\text { about problems in } \\
\text { my life }\end{array}$ & 0.137393 & 0.063723 & 0.160229 \\
\hline $\begin{array}{c}\text { Other people's pain } \\
\text { is very real to me }\end{array}$ & 0.386214 & 0.179353 & \\
\hline $\begin{array}{c}\text { It is important to } \\
\text { me to have power } \\
\text { over other people }\end{array}$ & 0.343434 & 0.301593 & \\
\hline $\begin{array}{l}\text { I have always been } \\
\text { an honest person }\end{array}$ & 0.122854 & & \\
\hline
\end{tabular}




\begin{tabular}{|c|c|c|c|}
\hline $\begin{array}{l}\text { When someone } \\
\text { reveals that s/he is } \\
\text { lonely, I want to } \\
\text { keep my distance } \\
\text { from him/her }\end{array}$ & 0.346376 & 0.260414 & 0.330101 \\
\hline $\begin{array}{l}\text { Before an important } \\
\text { decision, I ask } \\
\text { myself what my } \\
\text { parents would think }\end{array}$ & 0.06664 & 0.024248 & 1.748278 \\
\hline I have math anxiety & 0.074088 & 0.076468 & -0.03112 \\
\hline $\begin{array}{l}\text { I am a typically very } \\
\text { anxious }\end{array}$ & 0.398033 & 0.549235 & -0.2753 \\
\hline $\begin{array}{c}\text { I enjoy playing with } \\
\text { fire }\end{array}$ & 0.153313 & 0.182617 & -0.16047 \\
\hline $\begin{array}{l}\text { I am a hardcore } \\
\text { sports fan }\end{array}$ & 0.166932 & 0.115437 & 0.446082 \\
\hline $\begin{array}{c}\text { Politically speaking, } \\
\text { I consider myself to } \\
\text { be very } \\
\text { conservative }\end{array}$ & 0.664493 & 0.232371 & 1.85962 \\
\hline
\end{tabular}

Table 2. The variable-wise mean test $R^{2}$ in the models predicting twelve outcome variables from M-turk database and the differences between the model's accuracy using different set of predictors (Predictive Five Factors \Big Five Factors). 


\section{References}

1. Heider, F. (1958). The psychology of interpersonal relation. New York: Wiley.

2. Dweck, C. S. (2013). Self-theories: Their role in motivation, personality, and development. Psychology press.

3. Newmann, F. M. (1996). Authentic achievement: Restructuring schools for intellectual quality. Jossey-Bass.

4. Swann, W. B. (1984). Quest for accuracy in person perception: A matter of pragmatics. Psychological review, 91(4), 457.

5. Egisdóttir, S., White, M. J., Spengler, P. M., Maugherman, A. S., Anderson, L. A., Cook, R. S., ... \& Rush, J. D. (2006). The meta-analysis of clinical judgment project: Fifty-six years of accumulated research on clinical versus statistical prediction. The Counseling Psychologist, 34(3), 341-382.

6. Allport, G. W., \& Odbert, H. S. (1936). Trait-names: A psycho-lexical study. Psychological monographs, 47(1), i.

7. Cattell, R. B. (1943). The description of personality: Basic traits resolved into clusters. The journal of abnormal and social psychology, 38(4), 476.

8. McCrae, R. R., \& Costa, P. T. (1989). The structure of interpersonal traits: Wiggins's circumplex and the five-factor model. Journal of personality and social psychology, $56(4), 586$. 
9. Costa Jr, P. T., \& McCrae, R. R. (1990). Personality disorders and the five-factor model of personality. Journal of personality disorders, 4(4), 362-371.

10. Allik, I., \& Allik, I. U. (2002). The five-factor model of personality across cultures. Springer Science \& Business Media.

11. Benet-Martinez, V., \& John, O. P. (1998). Los Cinco Grandes across cultures and ethnic groups: Multitrait-multimethod analyses of the Big Five in Spanish and English. Journal of personality and social psychology, 75(3), 729.

12. John. O. P. (1990a). The "Big Five" factor taxonomy: Dimensions of personality in the natural language and in questionnaires. In L. Pervin (Ed.), Handbook of personality theory and research (pp. 66-100). New York: Guilford

13. McCrae, R. R., \& Costa Jr, P. T. (1986). Personality, coping, and coping effectiveness in an adult sample. Journal of personality, 54(2), 385-404.

14. John, O. P., \& Srivastava, S. (1999). The Big Five trait taxonomy: History, measurement, and theoretical perspectives. Handbook of personality: Theory and research, 2(1999), 102-138.

15. Gilead, Michael, Yaacov Trope, and Nira Liberman. "Above and beyond the concrete: The diverse representational substrates of the predictive brain." Behavioral and Brain Sciences (2019): 1-63

16. Fukunaga, K. (1990). Introduction to statistical pattern recognition, ser. Computer science and scientific computing. Boston: Academic Press.

17. Kosinski, Michal, David Stillwell, and Thore Graepel. "Private traits and attributes are predictable from digital records of human behavior." Proceedings of the national academy of sciences 110.15 (2013): 5802-5805.

18. Harari, Y. N. (2016). Homo Deus: A brief history of tomorrow. Random House.

19. Hough, L. M. (1992). The'Big Five'personality variables--construct confusion: Description versus prediction. Human performance, 5(1-2), 139-155.

20. Salgado, J. F. (2002). The Big Five personality dimensions and counterproductive behaviors. International journal of selection and assessment, 10(1-2), 117-125. 
21. Sibley, C. G., Osborne, D., \& Duckitt, J. (2012). Personality and political orientation: Meta-analysis and test of a Threat-Constraint Model. Journal of Research in Personality, 46(6), 664-677.

22. Gibney, E. 2018. The Scant Science Behind Cambridge Analytica's Controversial Marketing Techniques. Nature, March 29. doi:10.1038/d41586-018-03880-4

23. Mischel W. (1968). Personality and assessment. New York: Wiley.

24. Kosinski, M., Matz, S. C., Gosling, S. D., Popov, V., \& Stillwell, D. (2015). Facebook as a research tool for the social sciences: Opportunities, challenges, ethical considerations, and practical guidelines. American Psychologist, 70(6), 543.

25. Goldberg, L. R. (1992). The development of markers for the Big-Five factor structure. Psychological assessment, 4(1), 26.

26. Stillwell, D. J., \& Kosinski, M. (2015). myPersonality Project Website. Retrieved from http://mypersonality.org

27. Diener, E., Emmons, R. A., Larsen, R. J., \& Griffin, S. (1985). The Satisfaction With Life Scale. Journal of Personality Assessment, 49(1), 71-75.

28. Schwartz, S. H. (1992). Universals in the content and structure of values: Theory and empirical tests in 20 countries. In M. Zanna (Ed.), Advances in experimental social psychology (Vol. 25) (pp. 1-65). New York: Academic Press.

29. Baron-Cohen, S., \& Wheelwright, S. (2004). The empathy quotient: an investigation of adults with Asperger syndrome or high functioning autism, and normal sex differences. Journal of autism and developmental disorders, 34(2), 163-175.

30. Radloff, L. S. (1977). The CES-D Scale: a self-report depression scale for research in the general population. Applied Psychological Measurement, 1, 385-401.

31. Chen, L., \& Huang, J. Z. (2012). Sparse reduced-rank regression for simultaneous dimension reduction and variable selection. Journal of the American Statistical Association, 107(500), 1533-1545.

32. Winnicott, D. W. (2015). Deprivation and delinquency. Routledge.

33. Bowlby, J. (1988). Developmental psychiatry comes of age. The American journal of psychiatry. 
34. Arrindell, W. A., Sanavio, E., Aguilar, G., Sica, C., Hatzichristou, C., Eisemann, M., ... \& Kállai, J. (1999). The development of a short form of the EMBU: Its appraisal with students in Greece, Guatemala, Hungary and Italy. Personality and individual Differences, 27(4), 613-628.

35. Schönbrodt, F. D., \& Perugini, M. (2013). At what sample size do correlations stabilize?. Journal of Research in Personality, 47(5), 609-612.

36. Terwee, C. B., Bot, S. D., de Boer, M. R., van der Windt, D. A., Knol, D. L., Dekker, J., ... \& de Vet, H. C. (2007). Quality criteria were proposed for measurement properties of health status questionnaires. Journal of clinical epidemiology, 60(1), 34-42.

37. Wiggins, D. (1968). On being in the same place at the same time. The philosophical review, 77(1), 90-95.

38. Murray, H. A. (1938). Explorations in personality: A clinical and experimental study of fifty men of college age.

39. Jackson, D. N. (1965). Personality research form. Research psychologists Press, Incorporated.

40. Pratto, F., Sidanius, J., Stallworth, L. M., \& Malle, B. F. (1994). Social dominance orientation: A personality variable predicting social and political attitudes. Journal of personality and social psychology, 67(4), 741.

41. Saucier, G., \& Srivastava, S. (2015). What makes a good structural model of personality? Evaluating the Big Five and alternatives. Handbook of personality and social psychology, 3, 283-305. 